\title{
Prediction Model of Material Removal for Polishing Single Crystal Silicon by Cluster Magnetorheological Finishing with Dynamic Magnetic Fields Based on BP Neural Network
}

\author{
Qixiang Zhang, Mingliang Guo, Jiayun Deng, Jisheng Pan* \\ School of Electromechanical Engineering, Guangdong University of Technology, Guangzhou, China
}

Email address:

panjisheng@gdut.edu.cn (Jisheng Pan)

${ }^{*}$ Corresponding author

To cite this article:

Qixiang Zhang, Mingliang Guo, Jiayun Deng, Jisheng Pan. Prediction Model of Material Removal for Polishing Single Crystal Silicon by Cluster Magnetorheological Finishing with Dynamic Magnetic Fields Based on BP Neural Network. Engineering Science.

Vol. 5, No. 3, 2020, pp. 38-44. doi: 10.11648/j.es.20200503.13

Received: September 11, 2020; Accepted: September 27, 2020; Published: October 13, 2020

\begin{abstract}
Magnetorheological Finishing (MRF) is a new optical surface processing method, which has the advantages that good polishing effect, no subsurface damage, and suitable for complex surface processing. However, the interaction mechanism between the MRF pad and the workpiece is very complicated, so that the existing MRF material removal theoretical model is not accurate enough to establish the relationship between polishing parameters and material removal. In order to improve the processing efficiency and explore the material removal mechanism, a cluster magnetorheological finishing (CMRF) with dynamic magnetic fields method was proposed. Studying CMRF with dynamic magnetic fields material removal model is helpful to explain the removal mechanism more deeply, and improve the processing efficiency. In this study, the CMRF method was used to conduct a multi-factor orthogonal test on 2-inch single crystal silicon wafers. Based on the empirical Preston equation, the relationship between the machining gap and the polishing pressure was explained. Orthogonal experiments were done for a series of speeds, and obtaining the order of the influence of various factors on the average surface roughness $R a$ of the workpiece was: workpiece rotation speed $>$ polishing disk speed $>$ magnetic poles rotation speed $>$ oscillating speed; the material removal rate $(M R R)$ was: polishing disk speed $>$ workpiece rotation speed $>$ magnetic poles rotation speed $>$ oscillating speed. Then combining with the orthogonal experimental data, and taking the surface roughness $R a$ and $M R R$ as evaluation criteria, using Adam (Adaptive momentum) optimization algorithm to build a prediction model of $R a$ and $M R R$ for polishing single crystal silicon by CMRF with dynamic magnetic fields based on BP neural network. For the prediction result, $R a$ of the maximum error was $7.05 \%$, the minimum was $0.31 \%$; MRR of the maximum error was $10.22 \%$, the minimum was $1.32 \%$. Therefore, the feasibility of this model for predicting the results of CMRF was verified, and it laid a good foundation for the development of CMRF technology and its industrial application.
\end{abstract}

Keywords: Cluster Magnetorheological Finishing, Dynamic Magnetic Fields, Preston Equation, BP Neural Network, Prediction Model

\section{Introduction}

Magnetorheological Finishing (MRF) is a new optical surface processing method, which uses MRF fluid to generate magnetorheological effects under the action of magnetic fields to confine and clamp abrasives, thereby the abrasives change from a free state to a semi-fixed state [1-2]. MRF has the advantages that traditional polishing does not have, such as good polishing effect, no subsurface damage, and suitable for complex surface processing. MRF has been proven to be an efficient, ultra-smooth, and low-damage deterministic new optical surface processing technology [3-5]. However, in the classic MRF, the workpiece and the "polished ribbon" are in spot local contact, and the workpiece needs to be processed by trajectory scanning, which leads to low processing efficiency [6]. In order to improve the processing efficiency, a cluster magnetorheological finishing (CMRF) method in which small-sized magnetic poles are 
combined according to the cluster principle to form a large-area polishing pad is proposed. The method realizes the area contact between the polishing pad and the workpiece surface, so it can improve the processing efficiency and obtain nanoscale surface roughness [7]. On this basis, the static magnetic fields are transformed into dynamic magnetic fields by means of multi-poles eccentric rotation, which realizes real-time trimming of the CMRF pad. It is suitable for polishing single crystal silicon carbide, single crystal silicon, strontium titanate, indium phosphide and other semiconductor substrates, and can obtain surface roughness of nanometer or even angstrom level [8-10].

To improve the accuracy of CMRF, it is necessary to deeply understand its material removal mechanism, which is related to the choice of polishing parameters and the environment. In recent years, a variety of methods have emerged to study the material removal mechanism of MRF, including empirical models, finite element simulations, molecular dynamics, fluid mechanics models [11], etc. GROOTE et al. [12-13] polished glass materials with nano-diamond abrasives, and concluded that the material removal rate $(M R R)$ was linearly related to the physical and chemical properties of the workpiece, the size and concentration of abrasives, and the bonding strength of atomic bonds. SHOREY [14] based on the Preston equation and fluid lubrication theory found that the material removal efficiency of MRF was proportional to the shear stress of the workpiece, and the strength of the magnetic fields determined the yield stress. Therefore, the shape of the polished area was positively related to the size of the magnetic field. Shi et al. [15] calculated the numerical distribution of shear force and pressure in the polishing area through simulation, and fitted the surface of the influence factor of shear force and pressure on material removal, established a three-dimensional mathematical model of MRF material removal. The BK7 flat mirror with a diameter of $100 \mathrm{~mm}$ was tested and verified, the deviation of the peak removal rate was $3.36 \%$, and the deviation of the volume removal rate was $8.83 \%$.

However, the interaction mechanism between the CMRF pad and the workpiece is very complicated, so that the existing CMRF material removal theoretical model is not accurate enough to establish the relationship between polishing parameters and material removal. The development of BP (Back-propagation) neural network provides a precise method for exploring the material removal mechanism of CMRF. The neural network constructs a network model from experimental data through an adaptive learning method, thereby establishing the relationship between polishing parameters and material removal. Liu et al. [16] proposed an uneven surface polishing algorithm for industrial robots based on neural networks and genetic algorithms, and verified the effectiveness of the algorithm for predicting experimental results through polishing experiments on uneven surfaces. Guo et al. [17] established an intelligent decision-making system for grinding process through the improved BP neural network. Compared with the non-optimized BP neural network, the optimized neural network had significantly improved training accuracy and prediction accuracy. Yan et al. [18] established an abrasive water jet polishing BP neural network model, and carried out polishing experiments under the predicted incident angle and jet pressure. The minimum relative error between the actual value and the predicted value of the surface roughness of the aluminum alloy wheel was $0.92 \%$, the maximum was $2.89 \%$.

In this study, the CMRF with dynamic magnetic fields method was used to conduct a multi-factor orthogonal test on 2-inch single crystal silicon wafers. Based on the empirical Preston equation, the relationship between the machining gap and the polishing pressure in the CMRF with dynamic magnetic fields was explained. Combining with the orthogonal experimental data, and taking the surface roughness $R a$ and $M R R$ as evaluation criteria, using Adam (Adaptive momentum) optimization algorithm to build a prediction model of material removal for polishing single crystal silicon by CMRF with dynamic magnetic fields based on BP neural network. The feasibility of this model for predicting the results of CMRF was verified, and it laid a good foundation for the development of CMRF technology and its industrial application.

\section{Principles and Methods}

\subsection{Dynamic Magnetic Fields CMRF Processing Mechanism}

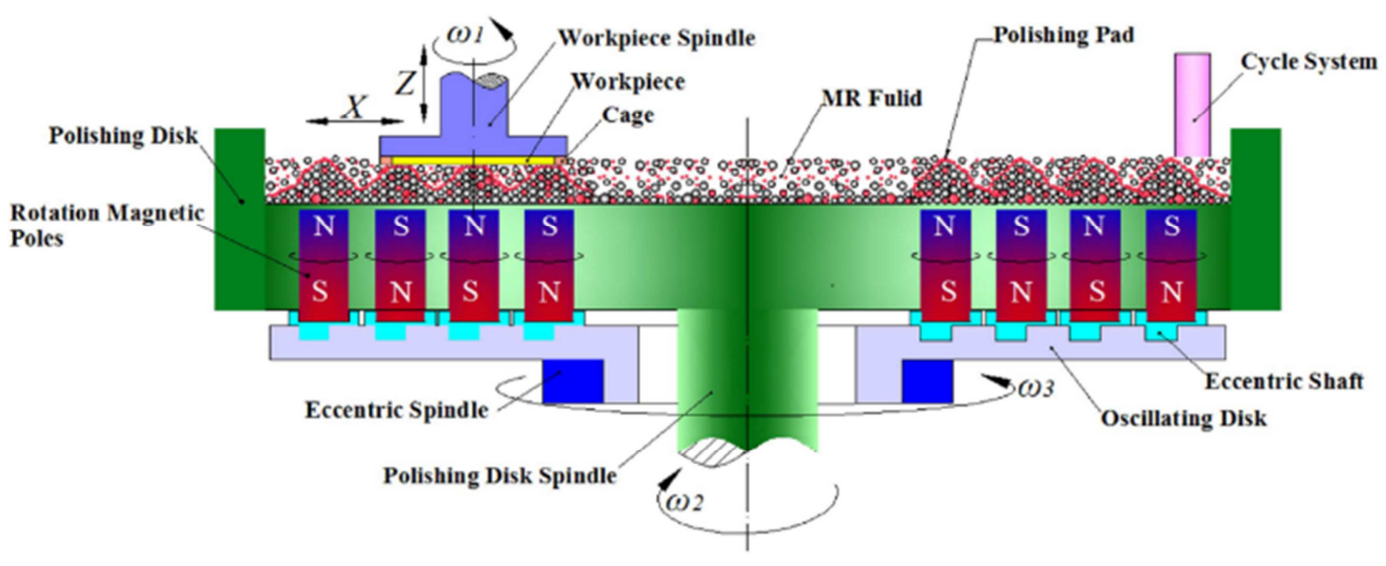

Figure 1. Diagram of CMRF with dynamic magnetic fields. 
Figure 1 shows the principle of dynamic magnetic fields CMRF. The magnetic poles were embedded in the polishing disk according to the cluster principle. The lower end of the magnetic poles was fixed on the eccentric shaft through an eccentric sleeve, the eccentric shaft was installed on the eccentric disk, and the lower end of the oscillating disk was installed on the polishing disk spindle. The magnetic poles and the eccentric shaft move eccentrically in the same direction. When the polishing disk spindle rotated, it would drive the eccentric disk and the eccentric shaft to move eccentrically in opposite directions. Since multiple magnetic poles had synchronous relative movement to the polishing disk, dynamic magnetic fields were formed on the surface of the polishing disk. When CMRF fluid was poured into the polishing disk, the magnetic particles were distributed along the lines of magnetic force to form a plurality of flexible polishing pads. Under the action of dynamic magnetic fields, the magnetic field lines changed in real time, and the CMRF fluid can form a new flexible polishing pad in real time. That is, when the polishing pad contacted with the workpiece for the first time, the polishing pad will be flattening, but the dynamic magnetic fields will restore the flattened polishing pad to its original shape quickly. So, the polishing pressure can be maintained constant, and the abrasives can be updated and reorganized continuously under the action of the dynamic magnetic field [19].

Figure 2 shows the experimental equipment of the dynamic magnetic fields CMRF. Multiple cylindrical permanent magnets (diameter $25 \mathrm{~mm} \times$ height $40 \mathrm{~mm}$ ) were placed under the polishing disk. The magnetic poles were driven by the dynamic magnetic field generator on the polishing disk surface, the dynamic magnetic fields were formed on the surface. The magnetic particles in the polishing liquid gathered along the direction of the magnetic field lines, thereby forming a dynamic flexible polishing pad on the polishing disk surface. The three single crystal silicon substrates were evenly attached to the lower surface of the workpiece spindle with paraffin. The workpiece spindle was fastened to the machine tool spindle through bolts, and the servo motion of the CNC milling machine was used. The synchronous rotation and machining gap were adjusted by controlling the $Z$ axis. The workpiece can oscillate and translate in the $\mathrm{X}$ or $\mathrm{Y}$ direction, and the oscillating displacement and oscillating speed can be controlled by CNC programming.

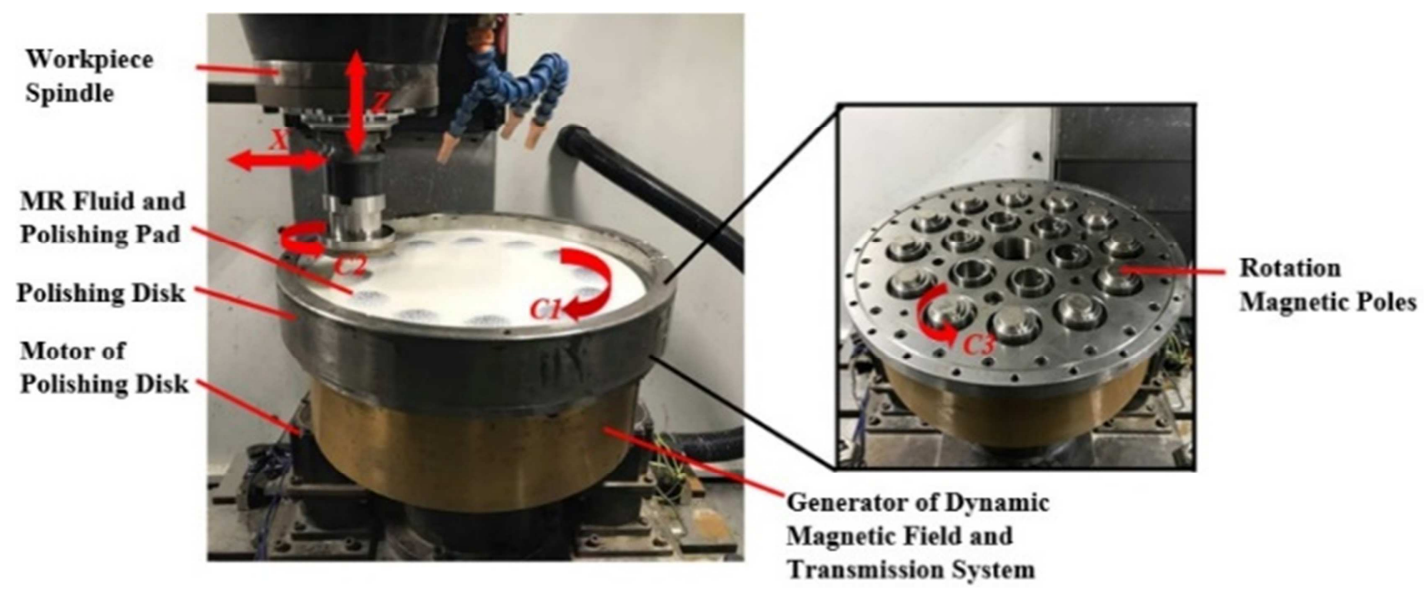

Figure 2. Experimental apparatus of CMRF with dynamic magnetic fields.

\subsection{Experimental Conditions}

The single crystal silicon substrates with a diameter of 2 inches $(\varphi 50.8 \mathrm{~mm})$ were used as the experimental material, and its original thickness was $450 \mu \mathrm{m}$, roughness $R a$ was 0.2 $\mu \mathrm{m}$. In the polishing process, the machining time, machining gap, magnetic poles eccentricity, oscillating mode, oscillating displacement and speed have a greater impact on the polishing effect and processing uniformity. The speed includes oscillating speed, magnetic poles rotation speed, polishing disk rotation speed, and workpiece rotation speed. These four parameters are related to each other, so orthogonal experiments were carried out for the four speeds. The process parameters required for CMRF are listed in Table 1. The CMRF fluid used in the experiment is made up of deionized water, carbonyl iron powder, abrasive, glycerin, etc. in a certain proportion.
Table 1. Process parameters and factor levels.

\begin{tabular}{ll}
\hline Process parameters & Level \\
\hline Machining time $(\mathrm{h})$ & 4 \\
Machining gap $(\mathrm{mm})$ & 1.0 \\
Magnetic poles eccentricity $(\mathrm{mm})$ & 6 \\
Oscillating method & $\mathrm{Y}$ \\
Oscillating displacement $(\mathrm{mm})$ & 40 \\
A: Oscillating speed $(\mathrm{mm} / \mathrm{min})$ & $200,400,600$ \\
B: Polishing disk speed $(\mathrm{r} / \mathrm{min})$ & $30,45,60$ \\
C: Magnetic poles rotation $\mathrm{speed}(\mathrm{r} / \mathrm{min})$ & $30,50,70$ \\
D: Workpiece rotation speed $(\mathrm{r} / \mathrm{min})$ & $250,350,450$ \\
\hline
\end{tabular}

\subsection{Evaluation Method of Machined Surface}

Taking 16 points equally divided along the surface of the single crystal silicon wafers as measurement points, as shown in Figure 3. Using an OLS4000 laser confocal microscope observes the micro-topography of the workpiece surface, and using Contour GT-X white light interferometer measures its surface roughness 
and observes its surface morphology. The measured average value was used as the surface average roughness of single crystal silicon. At the same time, a dial indicator (precision $0.1 \mu \mathrm{m}$ ) was measured the thickness change of single crystal silicon before and after processing, and the difference as the removal amount of the sample. The average removal amount of each point was taken as the average removal amount of the processed sample. The ratio of the average removal amount to the time as the average material removal rate.

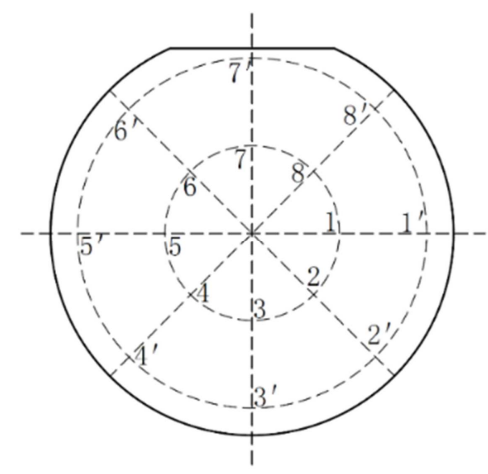

Figure 3. Distribution of sample measurement points.

\subsection{Experimental Results and Analysis}

To determine the influence law of four interrelated speeds on $R a$ and $M R R$ in the process of CMRF with dynamic magnetic fields, and obtain the optimal parameters combination on this basis. Set the process parameters according to Table 1 , carried out the $\mathrm{L}_{9}\left(3^{4}\right)$ orthogonal test with the four factors of oscillating speed, polishing disk speed, magnetic poles rotation speed, and workpiece rotation speed, and according to the method described in section 2.3 to detect the processing quality of single crystal silicon. The experimental scheme and results are shown in Table 2. From the range $R_{R a}$ and $R_{M R R}$ in Table 2, it can be seen that the order of the influence of various factors on the average surface roughness $R a$ of the workpiece is: workpiece rotation speed $>$ polishing disk speed $>$ magnetic poles rotation speed > oscillating speed; the MRR's is: polishing disk speed $>$ workpiece rotation speed $>$ magnetic poles rotation speed $>$ oscillating speed. It can be seen that the four speeds have important effects on $R a$ and $M R R$, so these four factors were used as input parameters of CMRF with dynamic magnetic fields material removal model.

Table 2. The experimental scheme and results.

\begin{tabular}{|c|c|c|c|c|c|c|c|}
\hline \multicolumn{6}{|c|}{ Experimental scheme } & \multicolumn{2}{|c|}{ Experimental results } \\
\hline \multicolumn{2}{|l|}{ Test number } & $\begin{array}{l}\text { Oscillating } \\
\text { speed/A }\end{array}$ & $\begin{array}{l}\text { Polishing disk } \\
\text { speed/B }\end{array}$ & $\begin{array}{l}\text { Magnetic poles } \\
\text { speed/C }\end{array}$ & $\begin{array}{l}\text { Workpiece } \\
\text { speed/D }\end{array}$ & $R a(\mathrm{~nm})$ & $M R R(\mu \mathrm{m} / \mathrm{min})$ \\
\hline \multicolumn{2}{|l|}{1} & 200 & 30 & 30 & 250 & 9.872 & 0.0225 \\
\hline \multicolumn{2}{|l|}{2} & 200 & 45 & 50 & 350 & 6.485 & 0.0536 \\
\hline \multicolumn{2}{|l|}{3} & 200 & 60 & 70 & 450 & 5.926 & 0.0512 \\
\hline \multicolumn{2}{|l|}{4} & 400 & 30 & 50 & 450 & 8.502 & 0.0374 \\
\hline \multicolumn{2}{|l|}{5} & 400 & 45 & 70 & 250 & 6.937 & 0.0482 \\
\hline \multicolumn{2}{|l|}{6} & 400 & 60 & 30 & 350 & 6.374 & 0.0508 \\
\hline \multicolumn{2}{|l|}{7} & 600 & 30 & 70 & 350 & 6.728 & 0.0479 \\
\hline \multicolumn{2}{|l|}{8} & 600 & 45 & 30 & 450 & 7.537 & 0.0453 \\
\hline \multicolumn{2}{|l|}{9} & 600 & 60 & 50 & 250 & 8.224 & 0.0472 \\
\hline \multirow{4}{*}{$\begin{array}{l}\text { the range of } \\
R a\end{array}$} & $\overline{K_{R a 1}}$ & 7.428 & 8.367 & 7.928 & 8.344 & & \\
\hline & $\overline{K_{R a 2}}$ & 7.271 & 6.986 & 7.737 & 6.529 & & \\
\hline & $\frac{K a L}{K_{P a 3}}$ & 7.496 & 6.841 & 6.530 & 7.322 & & \\
\hline & $R_{R a}$ & 0.225 & 1.526 & 1.398 & 1.815 & & \\
\hline \multirow{4}{*}{$\begin{array}{l}\text { the range of } \\
M R R\end{array}$} & $\overline{K_{M P P 1}}$ & 0.042 & 0.036 & 0.040 & 0.039 & & \\
\hline & $\frac{\bar{K}}{K_{M R R 2}}$ & 0.045 & 0.049 & 0.046 & 0.051 & & \\
\hline & $\overline{K_{M R R 3}}$ & 0.047 & 0.050 & 0.049 & 0.045 & & \\
\hline & $R_{M R R}$ & 0.005 & 0.014 & 0.009 & 0.012 & & \\
\hline
\end{tabular}

\section{Establishment of CMRF Material Removal Model}

\subsection{Theoretical Material Removal Rate Model}

Theoretical material removal model is an important factor for establishing a CMRF neural network model. CMRF is based on the rheological effect of MRF liquid for polishing. When external magnetic fields are applied, the viscosity of the MRF increases rapidly, and the shear stress also increases. The magnetic particles are magnetized gradually, which causing the magnetic particles to move along the direction of the magnetic force to form magnetic chains with a certain shear strength, and the abrasives are wrapped by the magnetic chains. The stacking and overlapping of magnetic chain strings becomes Bingham viscoelastic polishing pads with a certain shear strength [7]. When the lower surface of the workpiece and the upper surface of the polishing disc are in a reasonable machining gap and performing relative movement, the flexible polishing pad will form a certain pressure and shear speed on the surface of the workpiece, and then under the soft sliding action of the semi-fixed abrasive, the surface material of the workpiece is removed. The material removal model in this study is based on the Preston empirical equation, which can be further described as:

$$
M R R=K\left(P_{d}+P_{m}+P_{g}\right) V
$$

Where, $K$ is the Preston coefficient, $P_{d}$ is the hydrodynamic pressure, $P_{m}$ is the magnetization pressure generated by the magnetorheological effect, $P_{g}$ is the liquid buoyancy, and $V$ is the relative velocity of the MRF liquid 
and the surface of the workpiece in the polishing area.

It can be seen from formula (1) that under the condition of constant process conditions, only constant CMRF pressure can remove material of the workpiece continuously and stably. Under the condition that the surface of the workpiece is parallel to the surface of the polishing disc, theoretically the hydrodynamic pressure $P_{d}$ on the lower surface of the workpiece is negligible generally; the liquid buoyancy $P_{g}$ is much smaller than $P_{m}$, and can also be ignored. The pressure $P_{m}$ generated by the magnetorheological effect generally includes magneto strictive pressure and magnetizing pressure, but because the MRF liquid is incompressible, the magneto strictive pressure caused by volume change is zero. Therefore, it can be considered that the dynamic magnetic field polishing pressure is mainly the pressure $P_{m}$ that generated by the magnetorheological effect.

According to the research results of Zhang [20], the MRF pressure produced by the magnetorheological effect of spherical magnetic particles under the action of external magnetic fields is:

$$
\begin{array}{r}
P_{m}=\frac{3 \varphi \mu_{0}\left(\mu-\mu_{0}\right)}{\mu+2 \mu_{0}} \int_{0}^{H} H d H \\
H=\sum_{n=1}^{\infty}-A_{n} e^{-\beta_{n} Z}
\end{array}
$$

Among them, $A_{n}=\frac{2 B_{g} \sin \left(\beta_{n} e\right)}{\pi(2 n-1) \mu_{0}}, \beta_{n}=\frac{2 n-1}{2 a} \pi$

Where, $\varphi$ is a ratio of the magnetic particles occupied in the magnetorheological fluid, $\mu_{0}$ is the permeability of vacuum, $\mu$ is the permeability of the magnetic particles, $H$ is the magnetic field strength of the external magnetic field, $Z$ is the distance from the magnetic poles end face to the workpiece surface, $a$ is the total length of two magnetic poles placed side by side, $e$ is the width of the air gap formed by placing the soft magnetic material on the upper surface of the two magnetic poles side by side, and $B_{g}$ is the magnetic flux density passing through the air gap.

It can be seen from formula (2) that the pressure $P_{m}$ is positively related to the magnetic field strength $H$ of the external magnetic fields on the surface of the workpiece. When the pressure $P_{m}$ increases, on the one hand, the magnetic particles in the MRF fluid increase the holding force of the abrasive particles, on the other hand, the normal pressure of the abrasive in direct contact with the workpiece also increases, so as to achieve better MRR.

It can be seen from formula (3) that the absolute value of the applied magnetic field strength $H$ gradually increases with the reduction of the machining gap $Z$, thereby increasing the pressure $P_{m}$. Thus, when the machining gap $Z$ is constant, the magnetic field strength $H$ is a constant value, that is, the polishing pressure $P_{m}$ remains unchanged. In this case, the $M R R$ of CMRF with dynamic magnetic poles is mainly affected by the relative speed $V$. Taking into account the changes of four speeds involved in CMRF process, in order to explore the influence of speed changes on $R a$ and $M R R$, an orthogonal experiment with oscillating speed, polishing disk speed, magnetic poles rotation speed, and workpiece rotation speed as variables was designed. Taking the surface roughness $R a$ and $M R R$ as evaluation criteria, a material removal model based on BP neural network was established.

\subsection{Model}

Through training experimental data under different process parameters, the $R a$ and $M R R$ prediction model for polishing single crystal silicon by CMRF with dynamic magnetic fields based on BP neural network was established, which provides a basis for realizing the removal control of polishing single crystal silicon material. According to the results of the orthogonal experiment, taking the oscillating speed, polishing disk speed, magnetic poles rotation speed, and workpiece rotation speed as input parameters, and the surface roughness $R a$ and $M R R$ as output parameters, so the number of input layer nodes is 4 , and the output layer nodes is 2 . The hidden layer nodes are determined by formula (4):

$$
m=\sqrt{n+l}+a
$$

Where, $m$ is hidden layer nodes, $n$ is the input layer nodes, $l$ is the output layer nodes, and $\alpha$ is a constant between 1-10. So, the range of $m$ is range from 4 to 13 .

Although three layers BP network can map any n-dimensional to m-dimensional [21], after testing found that a three-layer network with hidden layer nodes of 13 requires tens of thousands of iterations to meet the requirements. Comparing the performance of each hidden layer nodes, finding four-layer network structure can achieve predicted results quickly, and finally determined that hidden layer nodes is 10 . Therefore, the network structure model for the $4 \times 3 \times 7$ $\times 2$, as shown in Figure 4 .

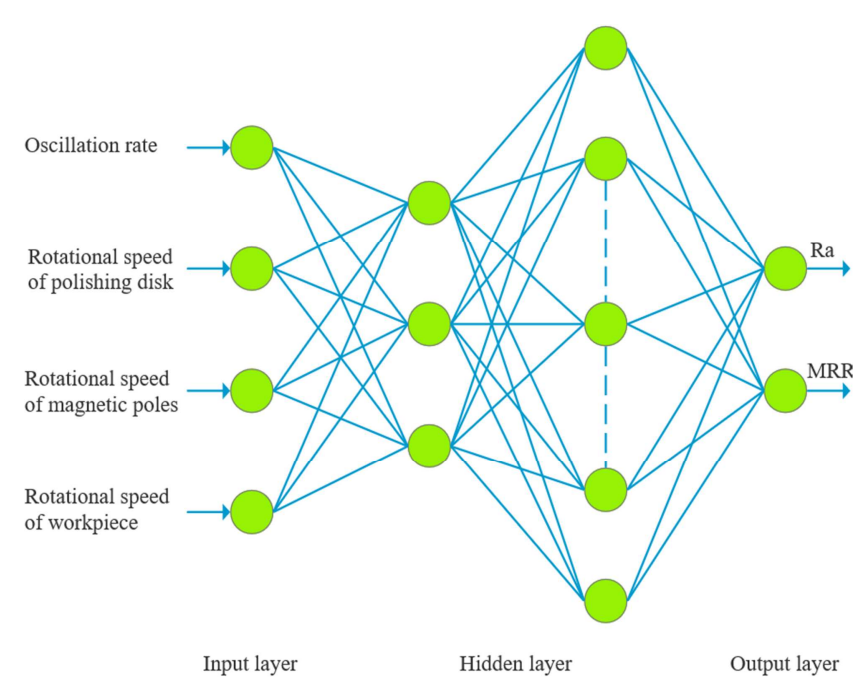

Figure 4. BP neural network structure.

In general, two classification problems, the hidden layer uses the tanh () function (5):

$$
\tanh (x)=\frac{\sinh (x)}{\cosh (x)}=\frac{e^{x}-e^{-x}}{e^{x}+e^{-x}}
$$

The output layer uses the sigmod () function (6) [21]:

$$
\operatorname{sigmod}(x)=\frac{1}{1+e^{-x}}
$$

In practical applications, the Adam method is better than 
other adaptive learning algorithms. Its convergence speed is faster, the learning effect is more effective, and it can correct the problems existing in other optimization techniques, such as the disappearance of the learning rate, the slow convergence or the parameter update with high variance leads to the loss function to fluctuate greatly [22]. Therefore, the input layer activation function used the tanh () function, the hidden layer activation function used the tanh () function, the output layer activation function used the sigmod () function, and the back-propagation function used the Adam optimization algorithm. Maximum number of iterations was set to 20000, initial learning rate was 0.001 . Stopping the calculation, when the error between the fitted value of the objective function and the true value was less than 0.0001 . Because the experimental data was less, all nine sets of data were used as training samples to train the network model, and two sets of data were selected randomly as test samples to test the network model. During the training period, the BP neural network corrected the weights continuously between the nodes through its independent learning ability, so that the model output results were approaching the sample data constantly, and stopping working when the training goal or the training numbers was reached. Through network calculation, when the number of iterations was 4000-7000, the required accuracy was reached. The mean square error (MSE) curve is shown in Figure 5, in the initial stage of training, the MSE value dropped significantly, and after 1000 iterations, the MSE value further dropped and gradually stabilized.

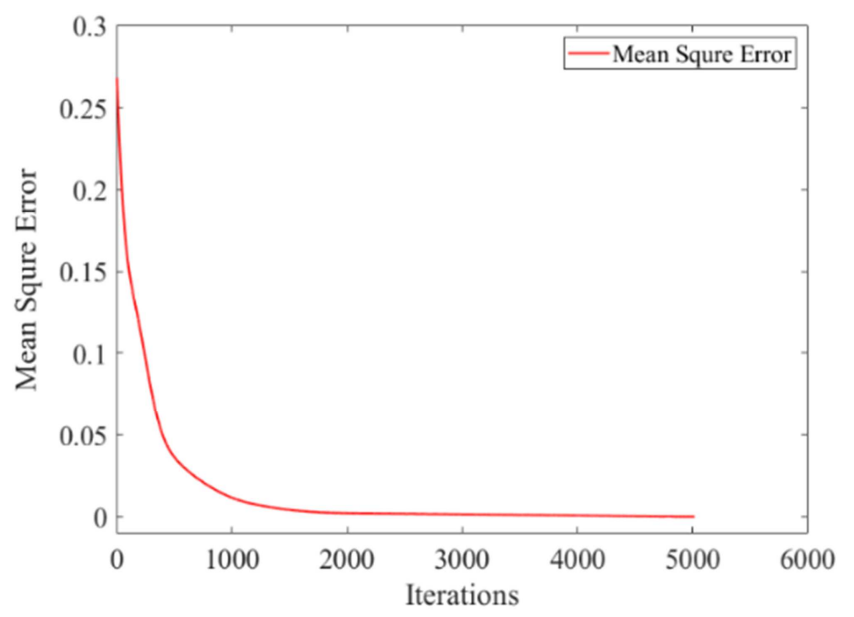

Figure 5. MSE changes during BP neural network training.

\subsection{Model Analysis}

Figure 6 shows the simulation results of the established network model are almost completely consistent with the measured results, and the fitting errors for $R a$ and $M R R$ are both within $1 \%$, which indicates that the model has a high degree of fitting, and can describe the relationship between the four process parameters and $R a$ and $M R R$ accurately.

In order to test the performance of the trained network model, the process parameters were input into the neural network, and then the error between the network output value and the experimental value was compared to determine whether the error meets the requirements. Table 3 lists the comparison between the measured values and predicted values of the BP neural network. For the prediction result, $R a$ of the maximum error is $7.05 \%$, the minimum is $0.31 \%$; $M R R$ of the maximum error is $10.22 \%$, the minimum is $1.32 \%$. The large errors in the prediction results are mainly due to the insufficient number of samples, which leads to fluctuations in the prediction results each time. If enough samples are used, the error of the prediction model can be further reduced. Therefore, it can be considered that within a certain error range, the established BP neural network model can predict effectively the $R a$ and $M R R$ of the CMRF with dynamic magnetic fields.
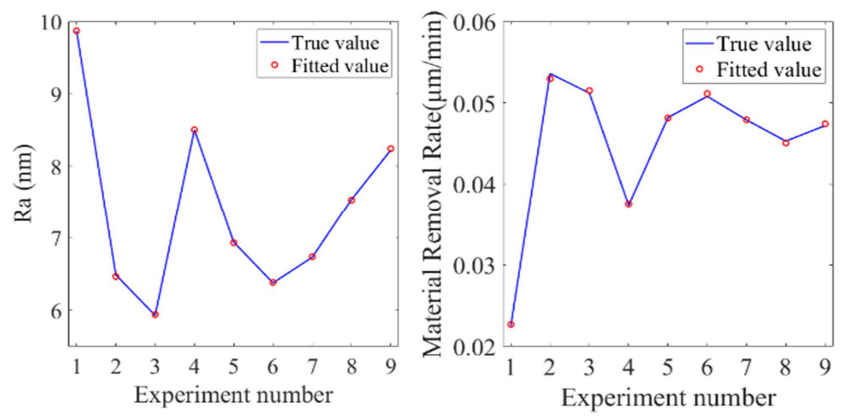

Figure 6. Comparison of simulation results and measured results.

It should be noted that the established BP neural network model is not a clear functional relationship, only a network structure. The $R a$ and $M R R$ prediction models trained by this BP network structure overcome the problem, that traditional theoretical models cannot describe accurately the complex nonlinear mapping relationship between process parameters and experimental targets, and provide new ideas for optimizing the polishing process.

Table 3. Comparison between measured values and predicted values.

\begin{tabular}{lllllll}
\hline & \multicolumn{3}{c}{ Measured value } & \multicolumn{4}{c}{ Predictive value } \\
\cline { 2 - 7 } & $\boldsymbol{R} \boldsymbol{a}$ & $\boldsymbol{M} \boldsymbol{R} \boldsymbol{R}$ & $\boldsymbol{R} \boldsymbol{M}$ & Error & $\boldsymbol{M R} \boldsymbol{B}$ & Error \\
\hline 1 & 9.872 & 0.0225 & 9.3218 & $5.57 \%$ & 0.0248 & $10.22 \%$ \\
2 & 6.485 & 0.0536 & 6.0293 & $7.03 \%$ & 0.0523 & $2.43 \%$ \\
3 & 5.926 & 0.0512 & 5.9444 & $0.31 \%$ & 0.0521 & $1.76 \%$ \\
4 & 8.502 & 0.0374 & 8.2112 & $3.42 \%$ & 0.0347 & $7.22 \%$ \\
5 & 6.937 & 0.0482 & 6.8308 & $1.53 \%$ & 0.0493 & $2.28 \%$ \\
6 & 6.374 & 0.0508 & 6.0612 & $4.91 \%$ & 0.0515 & $1.38 \%$ \\
7 & 6.728 & 0.0479 & 6.2536 & $7.05 \%$ & 0.0492 & $2.71 \%$ \\
8 & 7.537 & 0.0453 & 7.3955 & $1.88 \%$ & 0.0459 & $1.32 \%$ \\
9 & 8.224 & 0.0472 & 8.3582 & $1.63 \%$ & 0.0485 & $2.75 \%$ \\
\hline
\end{tabular}

\section{Conclusion}

As a new type of optical surface processing method, the CMRF with dynamic magnetic fields has great research significance for semiconductor surface polishing. Therefore, studying its material removal model is helpful to explain the removal mechanism more deeply, and improve the processing efficiency. In this study, the CMRF with dynamic magnetic fields method was used to conduct a multi-factor orthogonal test on 2-inch single crystal silicon wafers. Based on the empirical Preston equation, the relationship between the machining gap and the polishing pressure in the CMRF with dynamic magnetic 
fields was explained. Orthogonal experiments were done for a series of speeds, obtaining the order of the influence of various factors on the average surface roughness $R a$ of the workpiece was: workpiece rotation speed $>$ polishing disk speed $>$ magnetic poles rotation speed $>$ oscillating speed; the $M R R$ was: polishing disk speed $>$ workpiece rotation speed $>$ magnetic poles rotation speed $>$ oscillating speed. Combining with the orthogonal experimental data, and taking the $R a$ and $M R R$ as evaluation criteria, using Adam optimization algorithm to build a prediction model of material removal for polishing single crystal silicon by CMRF with dynamic magnetic fields based on BP neural network. For the prediction result, $R a$ of the maximum error was $7.05 \%$, the minimum was $0.31 \%$; $M R R$ of the maximum error was $10.22 \%$, the minimum was $1.32 \%$. Therefore, the feasibility of this model for predicting the results of CMRF was verified, and it laid a good foundation for the development of CMRF technology and its industrial application.

\section{Acknowledgments}

This project is supported by National Natural Science Foundation (No. 52075102 and U1801259), Guangdong Basic and Applied Basic Research Foundation (No. 2019A1515011243), Foshan Science and Technology Innovation Project of China (No. 2018IT100242) and Guangdong Province Degree and Graduate Education Reform Project (No. 2018JGXM35).

\section{References}

[1] Liu, X., Lu, H., Chen, Q., Wang, D., \& Zhen, X. (2013). Study on the preparation and properties of silicone oil-based magnetorheological fluids. Materials and Manufacturing Processes, 28 (6), 631-636.

[2] Guo, C. W., Chen, F., Meng, Q. R., \& Dong, Z. X. (2014). Yield shear stress model of magnetorheological fluids based on exponential distribution. Journal of magnetism and magnetic materials, 360, 174-177.

[3] Bai Z, Yan Q, Lu J, \& Xu X (2014). Research on Abrasive Accommodate-sinking Effect of Cluster MR-effect Polishing Pad. China Mechanical Engineering, 25 (20), 2705-2710.

[4] Catrin, R., Neauport, J., Taroux, D., Cormont, P., Maunier, C., \& Lambert, S. (2014). Magnetorheological finishing for removing surface and subsurface defects of fused silica optics. Optical Engineering, 53 (9), 092010.

[5] Wang, Y., Zhang, Y., \& Feng, Z. (2016). Analyzing and improving surface texture by dual-rotation magnetorheological finishing. Applied Surface Science, 360, 224-233.

[6] Shi, F., Shu, Y., Dai, Y., Peng, X., \& Li, S. (2013). Magnetorheological elastic super-smooth finishing for high-efficiency manufacturing of ultraviolet laser resistant optics. Optical Engineering, 52 (7), 075104.

[7] Pan, J., \& Yan, Q. (2015). Material removal mechanism of cluster magnetorheological effect in plane polishing. The
International Journal of Advanced Manufacturing Technology, 81 (9-12), 2017-2026.

[8] Lu J, Sun S, Yan Q, \& Liao B. (2019). Effects of Abrasive Characteristics on the Cluster Magnetorheological Polishing of InP Wafer. Semiconductor Technology, (1), 7.

[9] Pan, J., Yu, P., Yan, Q., \& Li, W. (2017). An experimental analysis of strontium titanate ceramic substrates polished by magnetorheological finishing with dynamic magnetic fields formed by rotating magnetic poles. Smart Materials and Structures, 26 (5), 055017.

[10] Yu, P., Pan, J., \& Yan, Q. (2016). Magnetorheological finishing with tangential magnetic fields formed by the rotation of a magnetic pole. International Journal of Abrasive Technology, 7 (4), 307-320.

[11] Yan J, Shao Q, Lv B, Zhao P, \& Wang J. (2020). Review on Material Removal Model of Flexible Contact Polishing Based on Rheological Principle. Journal of Mechanical Engineering, (3), 22.

[12] DeGroote, J. E. (2007). Surface interactions between nanodiamonds and glass in magnetorheological finishing (MRF) (Doctoral dissertation, University of Rochester. Institute of Optics).

[13] DeGroote, J. E., Marino, A. E., Wilson, J. P., Bishop, A. L., Lambropoulos, J. C., \& Jacobs, S. D. (2007). Removal rate model for magnetorheological finishing of glass. Applied optics, 46 (32), 7927-7941.

[14] Shorey, A. B. (2001). Mechanisms of material removal in magnetorheological finishing (MRF) of glass.

[15] Shi F, Dai Y, Peng X, \& Song C. (2009). Three-dimensional material removal model of magnetorhe ological finishing (MRF). China Mechanical Engineering, 20 (06), 0-673.

[16] Liu Y, \& Zhao Y. (2019). Uneven Surface Polishing of Industrial Robot Based on Neural Network and Genetic Algorithm. Machine tool \& Hydraulics, 47 (21), 46-50.

[17] Guo J, Zhang F, Xu C, \& Zhu Y. (2020). Intelligent Decision System for Lapping Process of Soft and Brittle Workpiece Based on Genetic Algorithm and Neural Network. Surface Thechnology, 49 (04): 23-29+46.

[18] Yan L, Lei Y, Tan L, Wu Y, \& Zeng X. (2017). Research on Polishing Quality of Abrasive Water Jet Based on BP Neural Network. Coal Mine Machinery 38 (12), 133-135.

[19] Pan, J., Qiusheng, Y. A. N., Gao, W., \& Yu, P. (2018). U.S. Patent No. 10,118,269. Washington, DC: U.S. Patent and Trademark Office.

[20] Zhang F, \& Zhang X. (2000). Foundation of mathematics model of magnetorheological finishing. Optical Technology, 26 (2), 190-192.

[21] Zhu J. (2001). Non-classical mathematics for Intelligent systems. Huazhong University of Science and Technology Press.

[22] Lin W. (2019). Some Application of Auto-adapted Optimization Algorithms under the Proportional Hazards Model. (Doctoral dissertation, Wuhan University). 